\title{
La arquitectura de prismas minimalistas de Oscar Niemeyer: Hipótesis de análisis por tipos
}

\section{Oscar Niemeyer's architecture of minimalist prisms: Hypotheses of analysis by type}

\author{
DOI: 10.17981/mod.arq.cuc.26.1.2021.06
}

Artículo. Fecha de Recepción: 23/08/2020. Fecha de Aceptación: 01/12/2020.

\section{Luca Bullaro}

Universidad Nacional de Colombia. Bogotá. D.C. (Colombia)

lbullaro@unal.edu.co

Para citar este artículo:

Bullaro, L. (2021). La arquitectura de prismas minimalistas de Oscar Niemeyer: hipótesis de análisis por tipos. MODULO ARQUITECTURA CUC, 26, pp. 137-160, 2021. DOI: http://doi.org/10.17981/mod.arq.cuc.26.1.2021.06

\section{Resumen}

El articulo — resultado de la investigación denominada "La herencia arquitectónica de Oscar Niemeyer"- analiza y propone una clasificación tipológica de una serie de obras realizadas por el maestro brasileño, caracterizadas por un sistema de prismas minimalistas, a menudo rectangulares en planta, en los cuales reina el ángulo de noventa grados. Son proyectos cuya sencillez está justificada por razones de economía constructiva, de reducción del presupuesto y, en el caso de los proyectos de conjuntos urbanos, para generar tensión y contraste con los vecinos volúmenes curvos. En el curso de la investigación se seleccionaron diferentes proyectos con características similares, se trazó una evolución de los varios esquemas, y se llegó a la proposición de una clasificación tipológica. Esta clasificación puede ayudar la comprensión del método de trabajo multifocal del maestro, y puede abrir nuevas trazas para ulteriores investigaciones sobre el perfeccionamiento metodológico que cumplió a lo largo de su carrera uno de los grandes maestros de la Modernidad de América Latina.

Palabras claves: Oscar Niemeyer; Modernidad de Brasil; prismas puros; minimalismo arquitectónico; monumentalidad moderna
Abstract

The article -result of the research called "The architectural heritage of Oscar Niemeyer" - analyzes and proposes a typological classification of a series of works by the Brazilian master, in which the passion for fluid spaces is not a basis for the genesis of the spatial system: they are projects whose simplicity -the architectural plants are always rectangular- is justified for reasons of constructive economy, budget reduction and, in the case of urban projects, to generate tension with neighboring curved volumes. Different projects with similar characteristics were selected, an evolution of the various schemes was traced, and a typological classification was proposed. This classification can help the understanding of the Niemeyer multifocal work method, and can open new traces for further research on the methodological improvement that one of the great teachers of Latin American Modernity.

Keywords: Oscar Niemeyer; Brazilian modernity; architectural boxes; architectural minimalism; modern monumentality 


\section{INTRODUCCIÓN}

El ángulo recto no me atrae, ni la línea recta, dura e inflexible, creada por el hombre. Lo que me atrae es la curva sensual y libre, la curva que encuentro en las montañas de mi país, en la mujer favorita, en las nubes del cielo y en las olas del mar, las curvas que componen todo el universo (...).

Paradójicamente, a pesar de esta reflexión una de las más famosas pronunciadas por Niemeyer - existe una serie de obras realizadas por el maestro dentro y fuera de su país natal, en las cuales la pasión por los espacios fluidos no ha sido fundamental en la definición del sistema espacial arquitectónico. Nos referimos a un conjunto de edificios de extrema sencillez, en los cuales Niemeyer aplica una "geometría pura", de tipo euclidiano, definida a través de un conjunto de prismas en los cuales el ángulo de noventa grados es protagonista, el color blanco uniforma las paredes revocadas, y las grandes superficies vidriadas y modulares relacionan de forma contundente los espacios interiores con los exteriores.

Son obras pensadas a veces como parte de un conjunto de edificios definido por el mismo arquitecto: en correspondencia directa con otros volúmenes que siguen reglas geométricas de matriz similar; o también en relación de dinámico contraste con vecinos cuerpos arquitectónicos formalmente predominantes, cubiertos a veces con grandes cupulas o bóvedas - como en el caso del Memorial de América Latina de Sao
Paulo- o caracterizados por sistemas de curvas y contracurvas, como por ejemplo en la marquesina central del parque Ibirapueira.

La fase inicial de la pesquisa se desarrolló a partir del análisis de algunas obras realizadas por Niemeyer que se seleccionaron, analizaron y agruparon a partir de algunas características en común, como por ejemplo la planta rectangular y el predominio geométrico del ángulo de noventa grados. Se investigaron luego los cambios y las transformaciones formales de este tipo de edificios dentro de la evolución metodológica de la poética del maestro, se extrapolaron unas reglas y se propuso una clasificación tipológica a partir de los análisis previos.

\section{Las interpretaciones criticas}

Bruno Zevi (1971) afirma que Niemeyer:

[...] posee dos almas creativas: a un lado geometrismo riguroso: prismas muy largos que se contraponen a la orgia de la naturaleza; al otro lado pinceladas sinuosas, abandono al paisaje, reconocimiento de un programa socialmente evasivo, alegre. (...) su metodología se basa en estas dos almas coexistentes: edificios como prismas sencillos, volúmenes aislados, independientes, autosuficientes, y cuerpos de conexión de gran tamaño, marquesinas dibujadas fluentemente, a mano alzada (p. 1).

La primera alma morfológica de Niemeyer - que se va repitiendo con importantes variaciones a lo largo de su carrera- deriva de una reinterpretación tropical de los "volúmenes puros" que Le Corbusier describe en "Hacia una 
arquitectura" (Bullaro, 2014), y también de la fascinación por las sencillas "cajas de vidrios" de Mies van Der Rohe. ¿Porque el maestro brasileño utiliza reiteradamente este sistema de prismas minimalistas? Los motivos son diferentes. Intentamos enumerar y explicitar algunos:

1. Aumenta la economía constructiva y reduce el presupuesto.

2. Facilita y acelera el proceso de construcción.

3. Genera tensión y contraste con las superficies cóncavos-convexas de edificios vecinos.

4. Facilita establecer una precisa jerarquía en el caso de conjunto de predios.

5. Evita una excesiva complexidad visual en los sistemas urbanos (que se generaría al utilizar morfologías complejas en la mayoría de los volúmenes).

6. Confiere importancia al sistema estructural y modular de los edificios.

7. Establece un contraste visual con los sistemas naturales vecinos (como por ejemplo en el caso del hospital de Rio en relación a la adyacente montaña del Corcovado).

La historiadora brasileña Ruth Verde (2012) afirma que los proyectos de los años cincuenta para Sao Paulo - aquellos desarrollados por el parque Ibirapueira - están a la base de este tipo de reducción y de simplificación formal.

El mismo Niemeyer atribuye a la experiencia para la construcción de Brasilia este "cambio" en su estrategia de proyecto:
Mi arquitectura - escribe- se vuelve en Brasilia más libre y más rigurosa: (...) rigurosa por la preocupación de mantenerla dentro de los limites regulares y definidos (...) Mi preocupación fue de caracterizarla a través de la estructura, permitiendo que, una vez terminada la estructura, la arquitectura ya estaba presente (Niemeyer, 1978).

Kenneth Frampton (2009), entusiasta de las obras más sensuales realizadas en los años cuarenta en Pampulha, interpreta en clave de "reacción neoclásica" los cambios metodológicos y morfológicos en la poética del maestro:

La obra de Niemeyer - escribe Framptonse hizo cada vez más simplista y monumental tras la publicación de los primeros esbozos para Chandigarh (....). Niemeyer rompió con la funcionalidad informal sobre las cual se habían basado sus fluidas formas planas para concentrarse en la creación de la forma puras, es decir, para acercarse más a la tradición neoclásica. Cabe fechar esta ruptura junto con su proyecto de 1955 para un museo de arte moderno en Caracas, donde propuso el uso impresionante de una pirámide invertida (...) señalando un retorno a los absolutos clásicos (p. 1).

En el texto Depoimento, publicado en las páginas de la revista brasileña "Modulo", Niemeyer (1958) confirma en parte el análisis de Frampton, pero subraya la importancia que este nuevo minimalismo arquitectónico aporta a la relación espacio-estructura, y la justifica también a través de la búsqueda de unidad y armonía entre edificios vecinos: 
Las obras de Brasilia marcan, junto con el proyecto del Museo de Caracas, una nueva etapa en mi trabajo profesional. Etapa que se caracteriza por una búsqueda constante de concisión y pureza, y una mayor atención a los problemas fundamentales de la arquitectura (...) Me interesé por las soluciones compactas, simples y geométricas; los problemas de jerarquía y carácter arquitectónico; las comodidades de unidad y armonía entre edificios (Niemeyer, 1958).

Verde (2012) confirma la interpretación del maestro carioca, escribiendo: "El cambio de rumbo en la obra de Niemeyer venía ocurriendo desde hace algunos años, en un período de transición que poco a poco consolida una nueva posición arquitectónica, que se despliega en las obras monumentales de Brasilia" (párr. 8).

El arquitecto Mendes da Rocha reitera la importancia de esta mitología de proyecto para la definición de conjuntos urbanos, y subraya también la influencia que Niemeyer generó en las nuevas generaciones de arquitectos brasileños:

El parque Ibirapueira - afirma Mendes da Rocha- fue esencial para abrir nuestros horizontes sobre una cuestión fundamental en la arquitectura: la disposición espacial. Era como si Niemeyer nos dijera: atención, no solo está el edificio, sino también el espacio circundante, y como todo interactúa (Niemeyer, 2012).

William Curtis facilita un nuevo enfoque a la interpretación reductiva de Frampton, condicionada probablemente por consideraciones políticas típicas de los años setenta del siglo pasado, subrayando el rol positivo de la elegancia monumental en las obras representativas para la nueva capital: "His contributions -escribe Curtis - to the new national capital of Brasília, such as the presidential Palácio da Alvorada, show that he could handle questions of monumentality and state representation with great elegance” (Wainwright, 2012).

\section{La relacion con los maestros de la modernidad}

Algunos años después de la feliz experiencia en Pampulha, donde se realiza un conjunto de edificios en algunos puntos específicos de la orilla del lago, Niemeyer es invitado a desarrollar un sistema complejo de conjuntos de edificios primero en Sao Paulo, y después en Brasilia.

En la búsqueda de "unidad y armonía", Niemeyer parece seguir el ejemplo de sus maestros (Bullaro, 2014): podríamos citar algunas obras de Le Corbusier y de Mies que están a la base de esta reinterpretación tropical: la sencillez en planta y en fachada del Pabellón de Suiza en la Ciudad Universitaria de Paris, aquella de los edificios representativos del Capitolio de Chandigarh, de Le Corbusier, el minimalismo de la casa Tughendat y del Campus del IIT de Mies Van Der Rohe.

Es muy probable que, en el desarrollo de los diseños para Sao Paulo y Brasilia, tuvieron una intensa influencia los proyectos que Niemeyer dibujó estando al lado de Le Corbusier — cuando 
el maestro suizo residió varias semanas en Rio de Janeiro, en los años treinta del siglo pasado - como la sede del Ministerio de Salud y Cultura en Rio de Janeiro y el sistema de prismas puros de la Universidad de Rio.

Otra referencia importante puede ser también el proyecto - realizado al final de los años cuarenta siempre a partir de unas ideas morfológicas claras y sencillas de Le Corbusier - para la sede de las Naciones Unidas en Nueva York (Harris, 1987).

El tema del prisma minimalista ha tenido, y continúa a tener, un notable éxito en la arquitectura moderna y contemporánea de Brasil: pensamos por ejemplo en la caja suspendida del MASP de Lina Bo Bardi — del final de los años cincuenta - en São Paulo, y también en los proyectos más recientes de Mendes da Rocha.

En Brasilia, los prismas blancos de Niemeyer son las piezas básicas cuya disposición espacial genera un nuevo sistema urbano monumental, en consonancia con la representatividad requerida por la moderna capital del País.

Esta tipología de edificios minimalistas, de planta rectangular alargada, estaba pensada a menudo para acoger las funciones menos representativas y más utilitarias, dejando a las formas convexas o mistilíneas el protagonismo de los espacios simbólicos, como por ejemplo acontece en el eje representativo de la nueva capital, en el cual la repetición monótona de los bloques de los Ministerios tiene como contrapunto el protagonismo formal de la sede del Parlamento y de la Catedral.
En varios edificios de Brasilia - escribe Niemeyer- como el palacio de justicia y la sede del ministerio exteriores, mi preocupación fue desarrollar una arquitectura más sencilla, elegante e repetida, fácil de ser elaborada y aceptada por la grande mayoría. Sería como un momento de pausa y reflexión para comprender mejor la arquitectura más libre que prefiero (Niemeyer, 2000).

La misma estrategia había sido aplicada unos años antes en el Parque Ibirapueira de Sao Paulo, donde un conjunto de prismas minimalistas dialoga con la intensidad cuatridimensional de las curvas del OCA, y está ordenado dentro de un preciso marco espacial gracias a la sensualidad de la marquesina orgánica, que funciona como columna vertebral y eje de conexión entre las diferentes cofres sencillos y monumentales que acogen el sistema expositivo.

\section{Desarrollo}

La primera fase de la pesquisa se desarrolló a partir del análisis de algunas obras realizadas por Niemeyer que se seleccionaron, se analizaron y se reagruparon a partir de algunas características en común, como por ejemplo la planta rectangular y el predominio geométrico del ángulo de noventa grados.

En la segunda fase, siguiendo el ejemplo del historiador Roberto Segre, se propuso investigar los cambios y las transformaciones formales de este tipo de edificios dentro de la evolución metodológica de la poética del maestro (Segre, 2012): desde la primera obra realizada, bajo la guía 
de Lucio Costa y Le Corbusier - el edificio del Ministerio de Educación y Salud en Rio- hasta la sede Mondadori de Segrate, construida en las afueras de Milán al final de los años sesenta.

En la tercera etapa se extrapolaron unas reglas y se propuso una clasificación tipológica a partir de los análisis previos.

Las varias aplicaciones del mismo tipo, en sus diferentes declinaciones, demuestran que la arquitectura de Niemeyer no se reduce a un uso expresivo, o caprichoso, de formas curvas, sino que, dependiendo de los requerimientos funcionales, y de los diálogos formales con el contexto, se desarrolla una específica tipología que responde también a los deseos de los clientes, al paisaje circundante y a las relaciones con las arquitecturas vecinas.

Con el transcurso de los años se nota una atención siempre mayor del maestro carioca hacia la monumentalidad y la pureza formal. Los cofres de los Ministerios de Brasilia y de la sede Mondadori, por ejemplo, dan cuenta de esta transformación metodológica de su quehacer arquitectónico hacia un nuevo tipo de monumentalidad moderna minimalista.

Los prismas puristas tienen un poder de ordenación territorial sencilla, aplicada en gran escala, que define las pautas básicas del proyecto territorial caracterizado por la repetición de formas claras y por la modulación de los elementos geométricos que configuran plantas y fachadas: una rigurosa plataforma básica que confiere protagonismo a los pocos volúmenes estratégicos y morfológicamente más complejos y experimentales.

En este sentido aparece emblemático, en Brasilia, el conjunto pautado de los bloques de oficinas de los ministerios que, a través de un diseño extremadamente sencillo, alcanza un dialogo metafísico con la Plaza de los Tres Poderes: un sistema de pantallas minimalistas que generan ritmos espaciales reiterados - que recuerdan las visiones del pintor italiano Giorgio De Chirico - para dejar el papel protagónico a las dos grandes cúpulas blancas del Parlamento y del Senado, a los arcos del Ministerio de Justicia y a la blanca flor de la Catedral.

\section{El Parque Ibirapueira}

Protagonista del Parque Ibirapueira, gracias a su forma orgánica, es la grande marquesina blanca que conecta los varios volúmenes minimalistas del conjunto expositivo. Es el único elemento del conjunto que reinterpreta la típica grafía que Bruno Zevi (1982) define "a mano libera" que recuerda la sensual cubierta de la Casa do Baile de Pampulha, y también la concatenación cóncavo-convexa de las orillas del vecino lago.

El Auditorio desempeña también un papel significativo gracias a su forma pura de gran tamaño y a su dialogo con la cúpula del "Oca". Se diferencia netamente de los otros cuerpos arquitectónicos en relación a su función específica que define su novedosa morfología. 
El primer proyecto para el auditorio, desarrollado en los años cincuenta del siglo pasado, tenía un carácter muy innovador: las fachadas laterales estaban configuradas a partir de un sistema de triángulos blancos que tocaba el suelo en un solo vértice. La reducción al mínimo de los puntos de apoyos transmitía una poderosa sensación de liviandad y de suspensión (Papadaky, 1954).

El proyecto realizado años después -inaugurado en el 2005 - conserva algunos aspectos en común con el antiguo diseño, pero sin poseer la misma audacia espacial y estructural. En las fachadas laterales vuelve a aparecer la forma triangular (Figura 1). El frente principal, que alberga el acceso, es un plano inclinado y blanco - que rememora la colina artificial realizada en Chandigarh por Le Corbusier, y también la morfología del Teatro de Brasilia (ambos diseñados en los años cincuenta del siglo pasado)— en la cual una escultura metálica en forma de lengua roja, realizada por la artista brasileña Tomie Ohtake, conforma una marquesina simbólica que indica el acceso al hall principal (Serapiao, 2005).

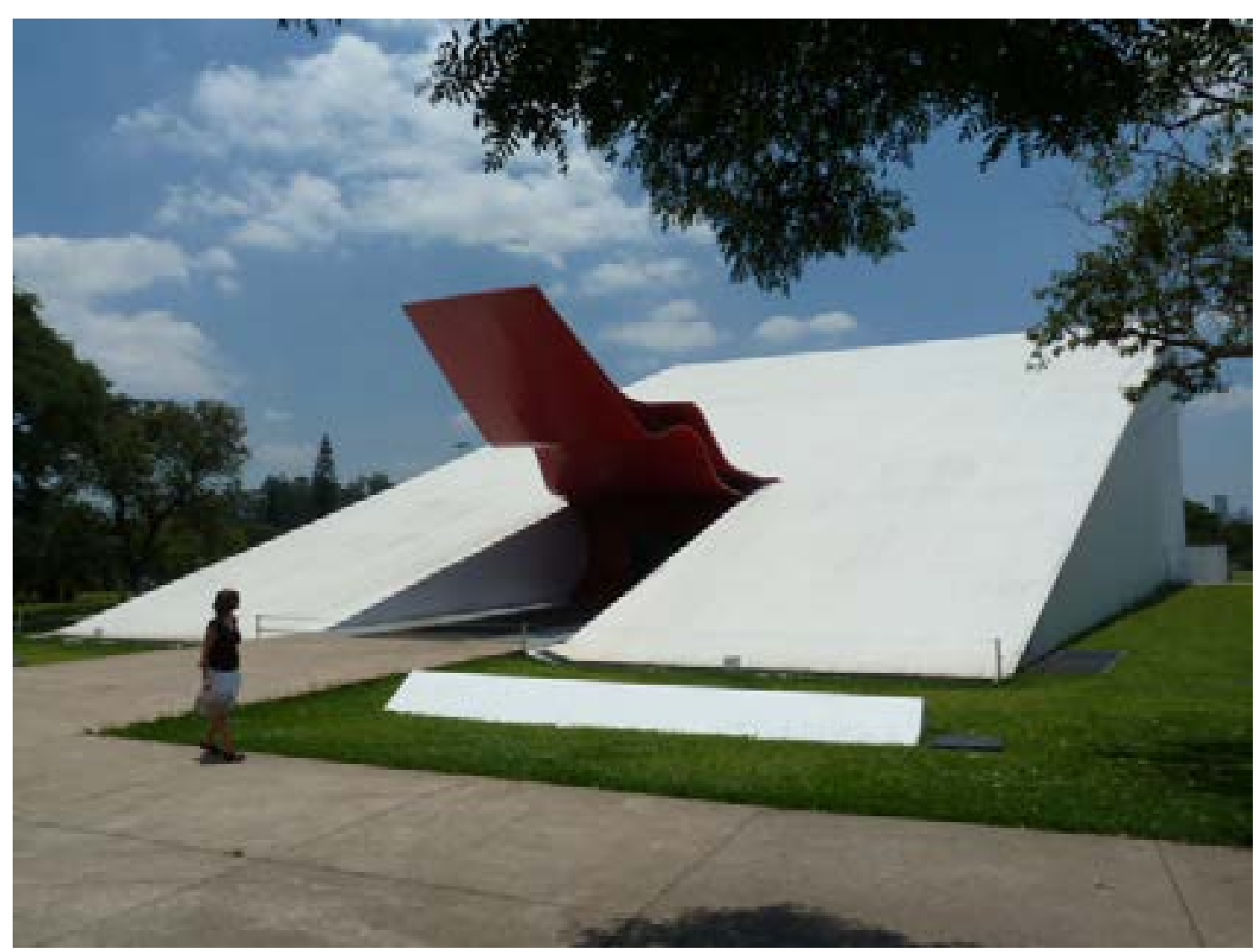

Figura 1. Auditorio en el parque Ibirapueira de São Paulo (Oscar Niemeyer).

Fuente: Foto de Luca Bullaro. 
Los volúmenes del Auditorio y del "Oca" so que protagonizan el conjunto (Figura 2). Podrian ser una representación metaforica de la hoz y el martillo, emblema que encarna la unión de los trabajadores -obreros y campesinos - usado como símbolo del comunismo, que Niemeyer apoyó con vigor a lo largo de toda su vida.

En los años cincuenta se realizaron en el parque tres pabellones de gran tamaño que se configuran como sencillos cofres de grandes dimensiones: volúmenes puros bajo la luz tropical donde, como en algunas arquitecturas de los años veinte y treinta de Le Corbusier, las sorpresas florecen en el espacio interior.

El edificio del Palacio de la Industria, por ejemplo, muestra una complejidad estra, por terior difícilmente reconocible al observar las minimalistas fachadas. El Palacio de los Estados y el Pabellon de las Naciones son los pabellones menores y están implantados con orientaciones distintas (Figu3). Posen dos pisos de 150 por 42 metros 3). Posten dos pis de 150 por 42 metros y una estructura caracterizada por generosos hormigón armado.

Figura 2. Relación entre el Oca y el Auditorio marquesina organica en el parque
Ibirapueira de Sã Pal Fuente: Google Maps $\odot$.

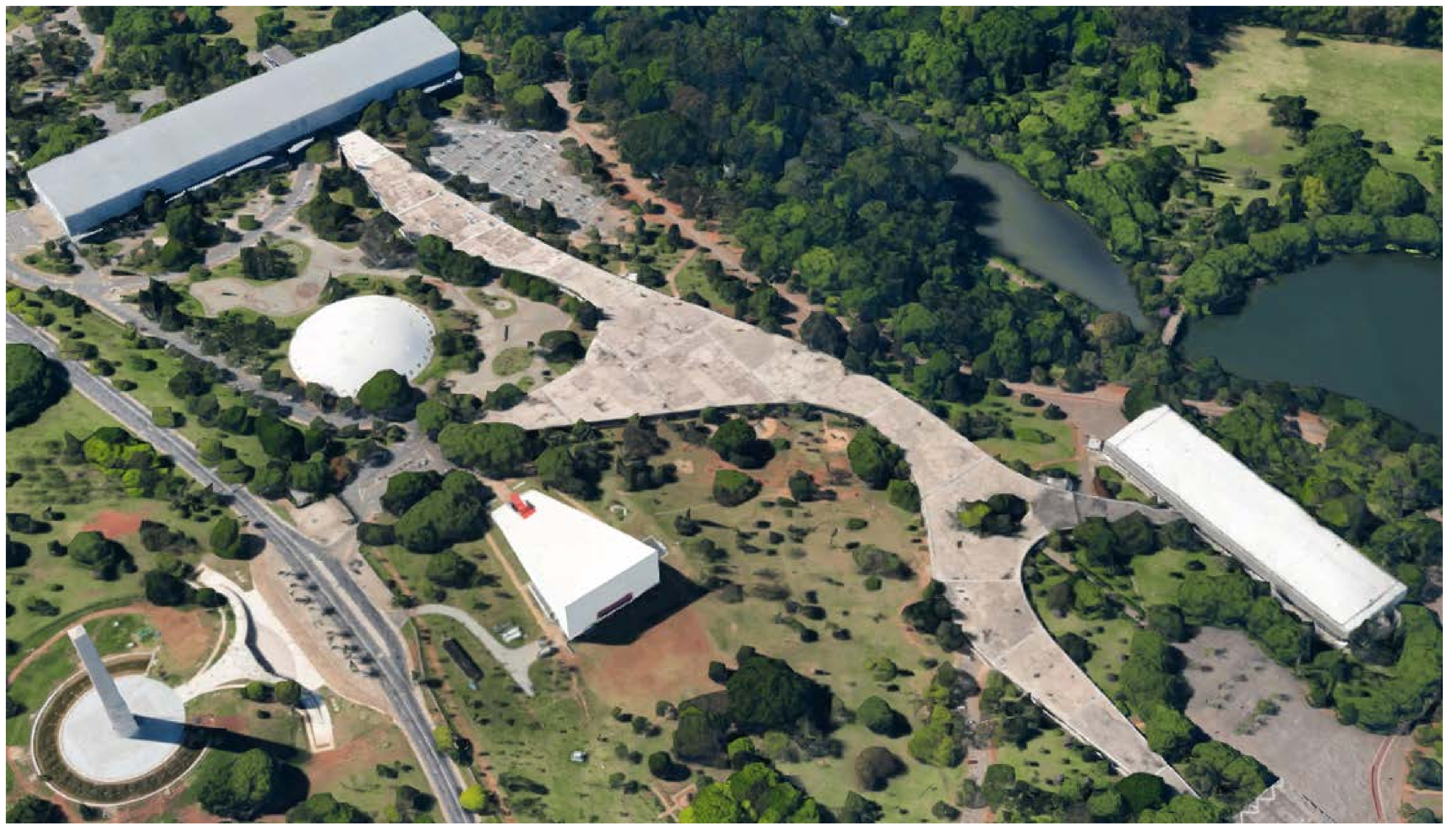




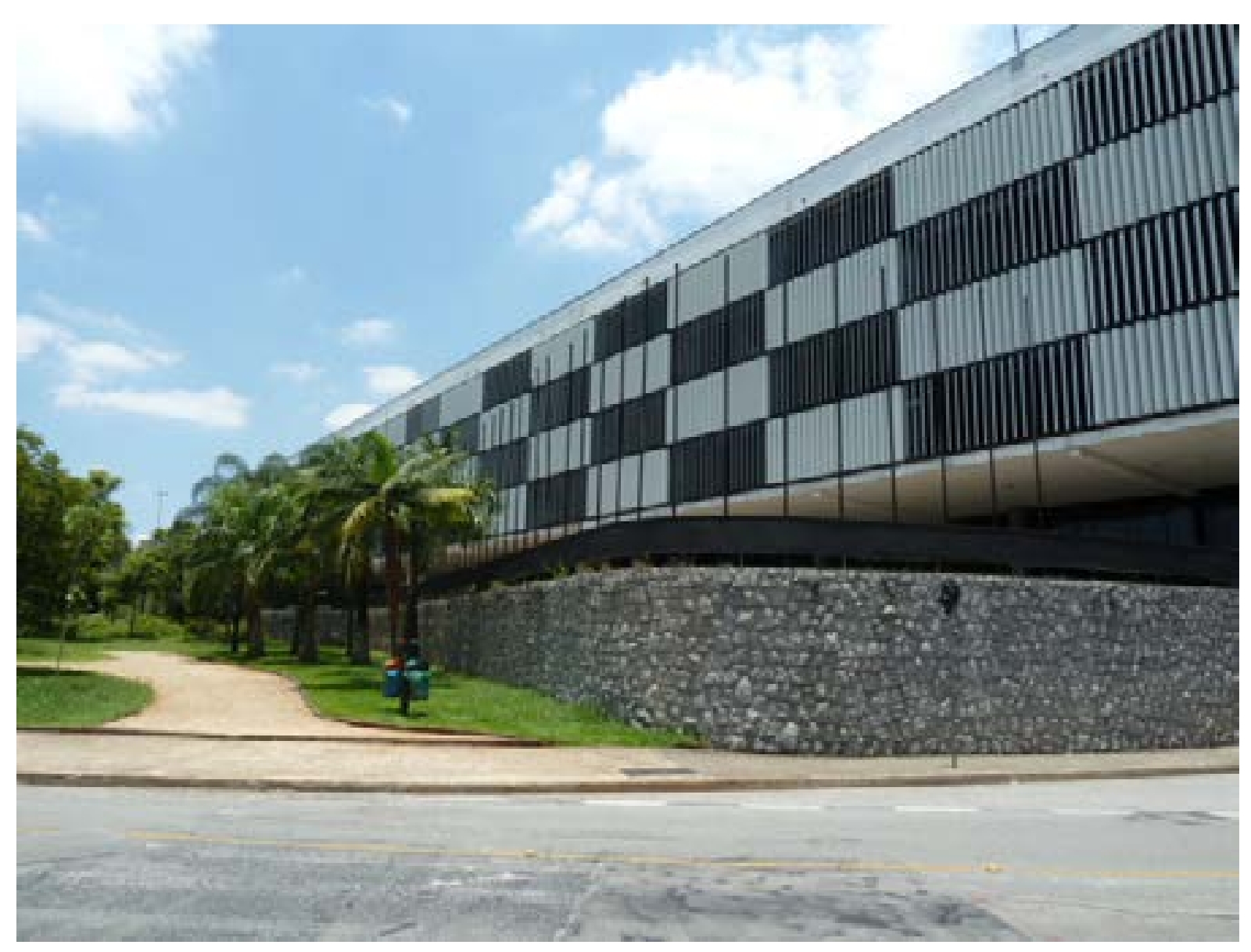

Figura 3. Pabellon de las Naciones. Parque Ibirapueira en São Paulo (Oscar Niemeyer).

Fuente: Foto de Luca Bullaro.

En el desarrollo del proyecto del conjunto Niemeyer vuelve a escoger como referente algunos temas de la poética de Le Corbusier, en la planeación de un sistema homogéneo y coherente en el cual todas las funciones expositivas están albergadas en unos espacios sencillos, configurados según las leyes geométricas del ángulo recto, y conectados gracias al juego sensual de la marquesina orgánica central.
En los pabellones se desarrolla un sistema de espacio central de triple altura, en el cual el perímetro interior de los forjados sigue líneas curvas que se repiten en las superficies sinusoidales de las barandillas, y se articulan gracias a los sistemas protagónicos de conexión vertical en una explosión formal que parece acompañar los movimientos de los usuarios y facilitar la contemplación dinámica de objetos y obras de arte. 
El Palacio de la Industria - hoy sede de la Bienal de São Paulo- es el pabellón de mayor dimensión, realizado en hormigón armado, con una malla estructural de diez por diez metros, una "fachada libre» vidriada que configura un perímetro puro que vibra en correspondencia de los quiebra-soles y de los corredores abiertos de la planta baja.

El interior está marcado por el sinuoso vacío protagonizado por la rampa que conecta los varios niveles. Existe un fuerte contraste entre la sobriedad de la fachada noroeste, protegida con brise-soleil de aluminio y la riqueza dinámica del interior, manifestada en el perímetro de los forjados de las plantas intermedias y en el contorno de las rampas. Los suelos, negros y brillantes, actúan como un espejo que refleja las curvas sinusoidales de los pasamanos blancos y los planos inclinados de los elementos de conexión vertical.

\section{El minimalismo monumental de Brasilia}

En Brasilia Niemeyer juega en repetidas ocasiones con composiciones espaciales de prismas puros, cuyas variaciones residen a menudo en el tratamiento de la piel exterior. En una de las fases de la investigación se propuso una clasificación de estas sub-tipologias según la caracterización de la envolvente, de en algunos casos sencilla (pan de verre), en otros casos doble (desarrolladas a partir de exigencias bioclimáticas), o monumental (a veces con el uso de grandes arcos en hormigón armado).

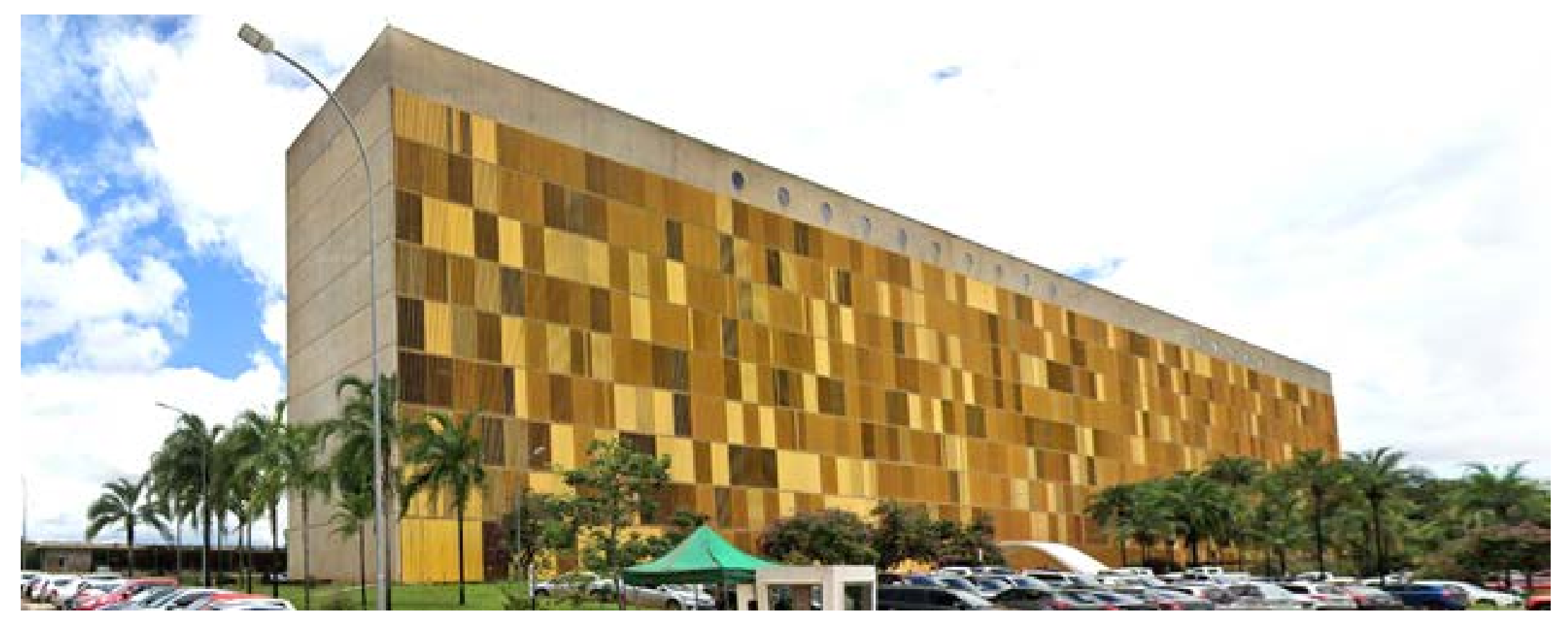

Figura 4. Edificio para las oficinas del Parlamento de Brasilia (Oscar Niemeyer).

Fuente: Google maps (C). 
El primer caso, más purista, se aplica a la modulación pautada del eje de los ministerios ficinas del Parlamento de Brasilia (Figura 4); el ultimo subtipo, más impon (Tigura , el ultim subtipo, más imponente y monumental, se registra en los edificios más emble-

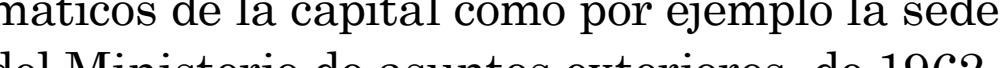

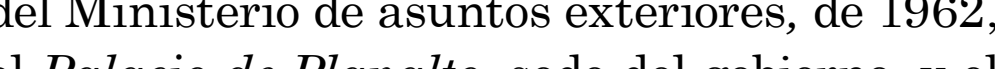
Triburio de Planatu, sede del gobierno, y el Tribunal Supremo Federal, que configuran el perímetro de la Plaza de los tres poderes, pro5).

En ambos casos el arquitecto entabla un juego espacial a partir del concepto de repetición, generando unas pautas espaciales de tipo musical, como preludio a la aparición de una arquitectura simbolica y monumental, segun la concepción urbanística y la disposición volumétrica planeada por Lucio Costa (1961) en el proyecto urbano general.

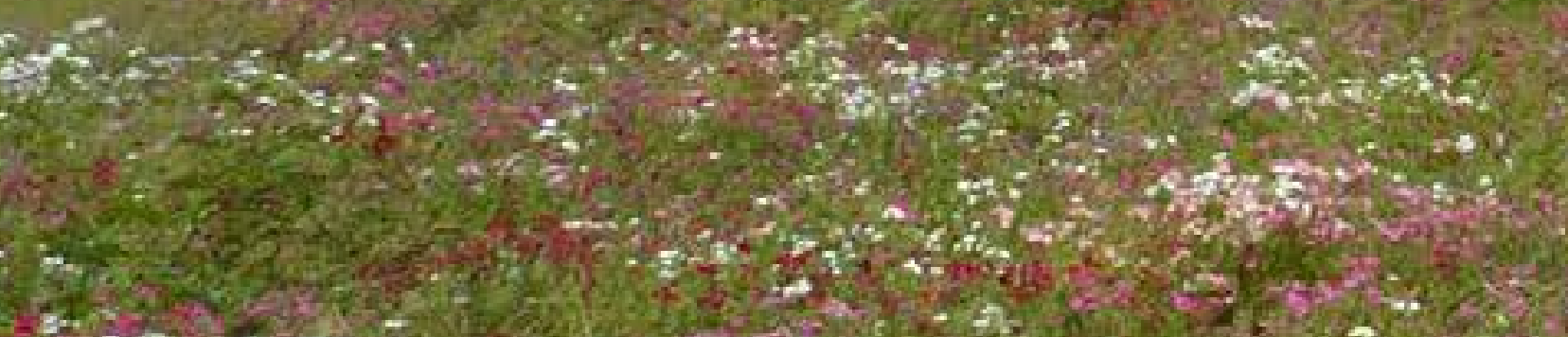
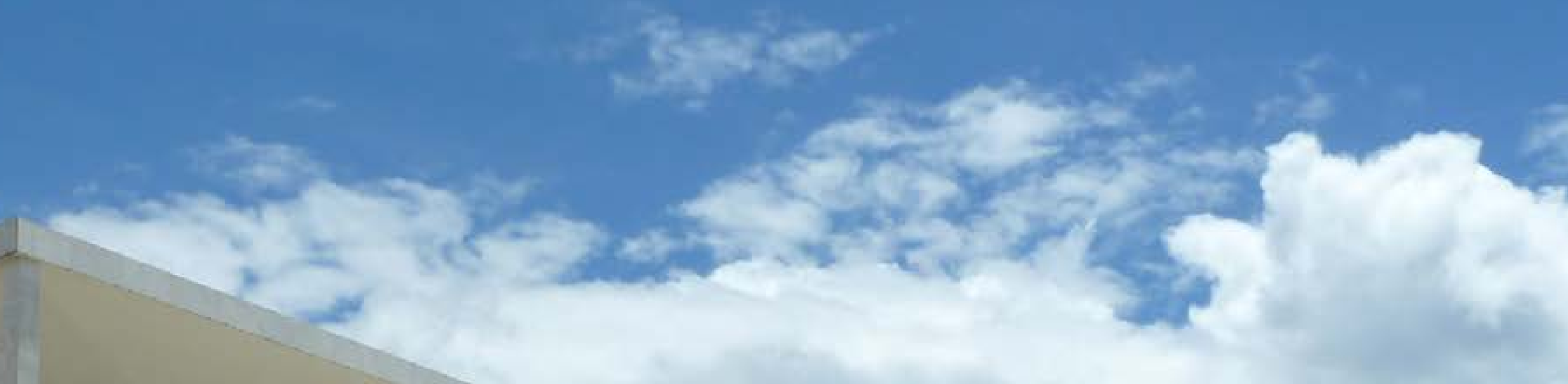

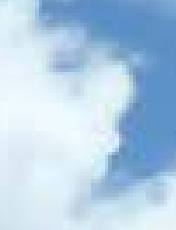
rata
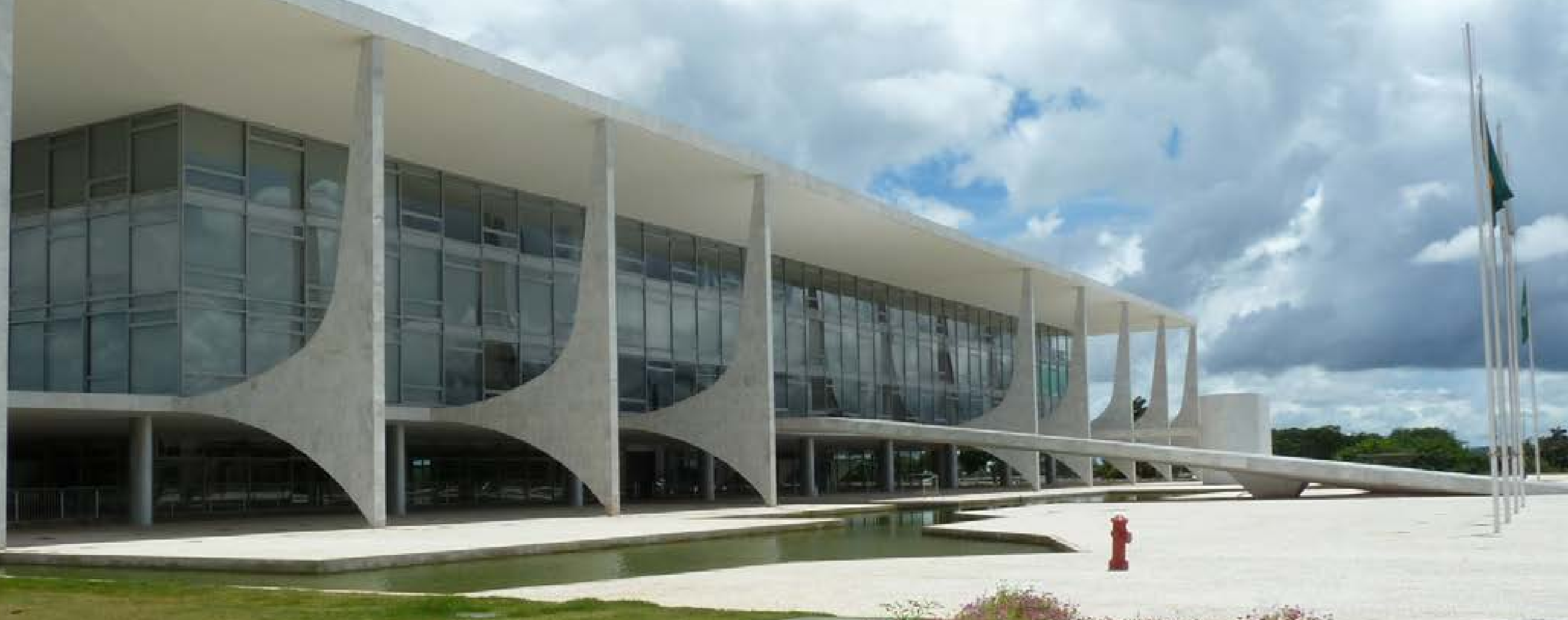

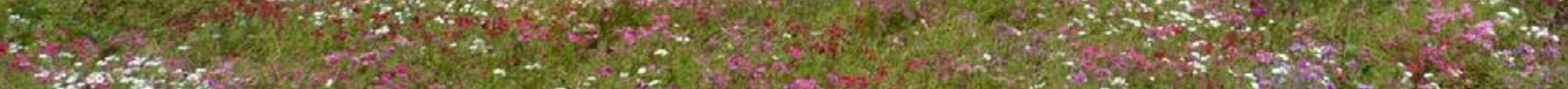


Los volúmenes más emblemáticos, de doble piel, presentan la cara interior, sencilla y vidio continus. En la me parana exterior de reinterpreton algunos carateres monumentales de la arquitectura áclica en forma de

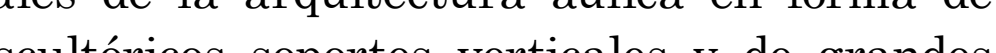
arcos rinterpretalos en clave más dinámica y modre en moderna nuevos materiales co e La fachada de la sede del

La fachada de la Ministerio de Asuntos Exteriores (Figura 6) está delineada por esbeltos pilotis que sostienen arcos monumentales en hormigón armado. Parece reinterpretarse en este caso y la memoria de la arquitectura imperial romana, y los experimentos de monumentalidad moderna que Le Corbusier aplica por ejemplo en el proyecto para la sede de la Corte suprema de Chandigard, la nueva capital del estado del Punjab, en la India (Figura 7)

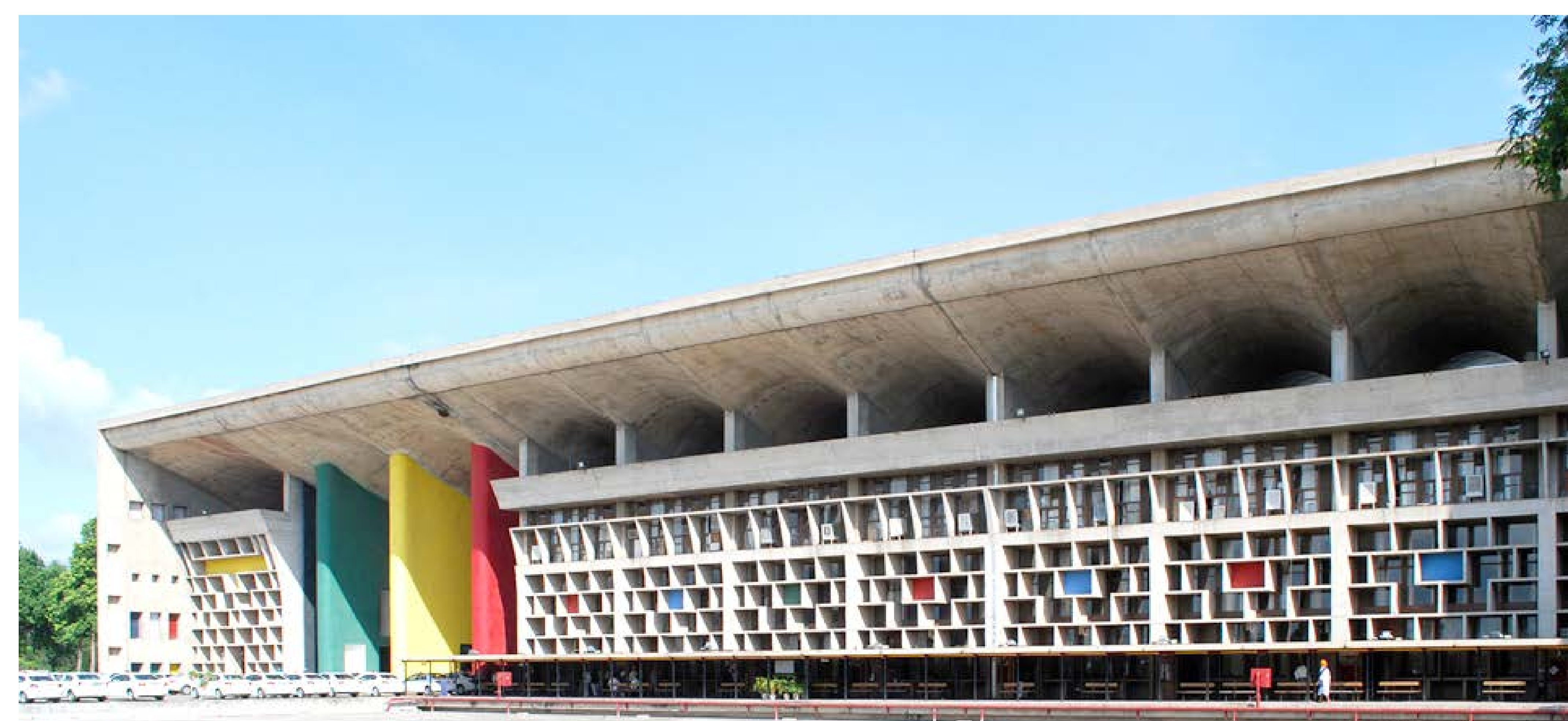

Figura 7. La sede del Tribuna
Federal de Chandigarh. Fuente: Sanyam Bahga, 201 
Escribe Niemeyer en el libro "Minha experiência em Brasília" publicado después de la nauguración de la ciudad: "mi preocupación era que defniera y caracterizat los edificios prinC de citerios de prilos inpescing Palcio Ducal, la Catedral de Char Ites, obras cacio obras que causan un impacto inde Chartihle por la (..). Sólo la belleza plastica actua y don (... ). Sólo la belleza plástica actúa y domina, como mensaje permanente de gracia y poesia" Niemeyer, 1960, p. 3).

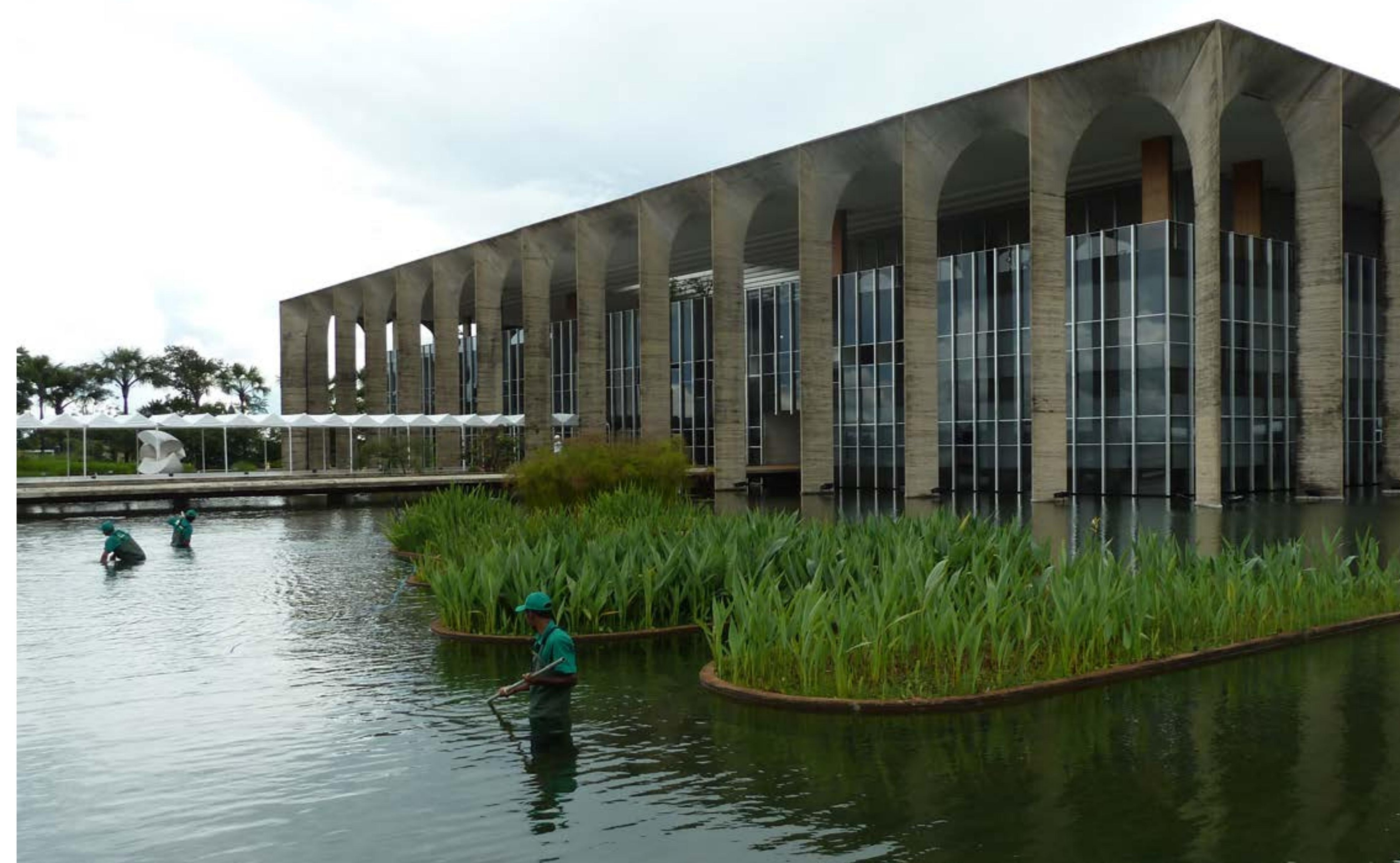


Otra serie de prismas minimalistas realizados por Niemeyer se encuentran fuera del territorio brasileño: volumenes suspendidos, cuerpos flotantes que generan amplios espacios abrigados en la planta baja: plazas cubiertas que los ciudadanos pueden utilizar y transformar cada día.

En Segrate, a pocos kilómetros de Milán, en Italia, Niemeyer intenta fusionar el tema de la caja suspendida con la reiteración de una fachada monumental para magnificar la actividad de Giorgio Mondadori, importante empresario italiano que, fascinado por el edificio del Ministerio de Asuntos Exteriores de Brasilia, solicita en 1968 el proyecto de la sede de la editorial según el mismo esquema morfológico (Figura 8).

La reinterpretación que se concibe de la imponente arquitectura de los grandes viaductos y de los acueductos romanos adquiere en esta obra una declinación novedosa: los arcos se hacen dinámicos, los ritmos musicales; los soportes no se imaginan siempre a la misma distancia entre sí, sino según unas pautas similares al sistema de "quiebra-soles musicales" experimentado por Le Corbusier en el Monasterio de La Tourette, en Francia.

Figura 8. La sede de la editorial
Mondadori en Segrate (Milano). Fuente: Foto de Luca Bullaro

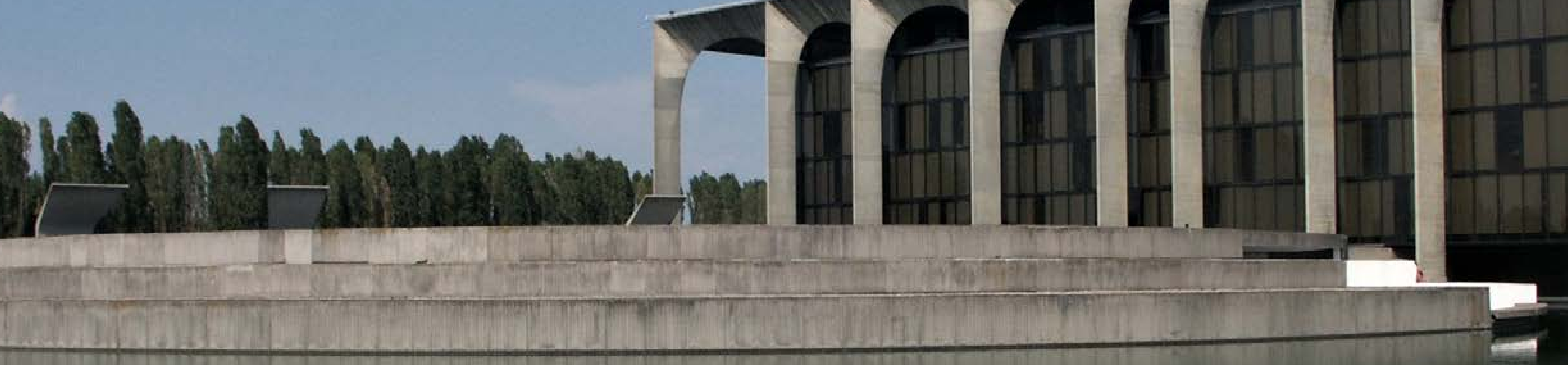


Las altas columnas sostienen el forjado de la cubierta que, gracias a un sistema de tensores, permite la suspensión de los forjados de los niveles de las oficinas y consiente la eliminación de los soportes internos en la planta baja: los únicos puntos de apoyo que tocan el suelo definen el perímetro exterior y están sumergidos en el espejo de agua oscuro, que parece multiplicarlos.

Transitando por la planta baja, abierta en su totalidad, la sensación espacial que la arquitectura transmite es novedosa, soñadora. La caja oscura, suspendida, completamente acristalada, parece flotar y el agua que lo rodea contribuye a crear con sus brillos y sus reflejos un sistema espacial y paisajístico impactante.
La sede italiana de FATA Engineering, realizada en 1975 en Pianezza, a las afueras de Torino, siempre en Italia, se desarrolla a partir de un sistema estructural de tipo similar a aquello utilizado en la sede Mondadori. En este caso el número de los soportes es extremadamente reducido - seis grandes pilares-que el noto ingeniero italiano Riccardo Morandi concatena, según el elegante esquema de Niemeyer, con la poderosa jácena perimetral y con los tensores en hormigón (Figura 9). Se crea un conjunto homogéneo que incluye elementos cargados a compresión y a tracción a través de la repetición de arcos en voladizo que sostienen los forjados de los varios pisos. "Fue la primera vez - declaró Morandi-que un trabajo me obligó a recorrer a todo lo que sabia sobre el hormigon armado (Niemeyer, 1978).

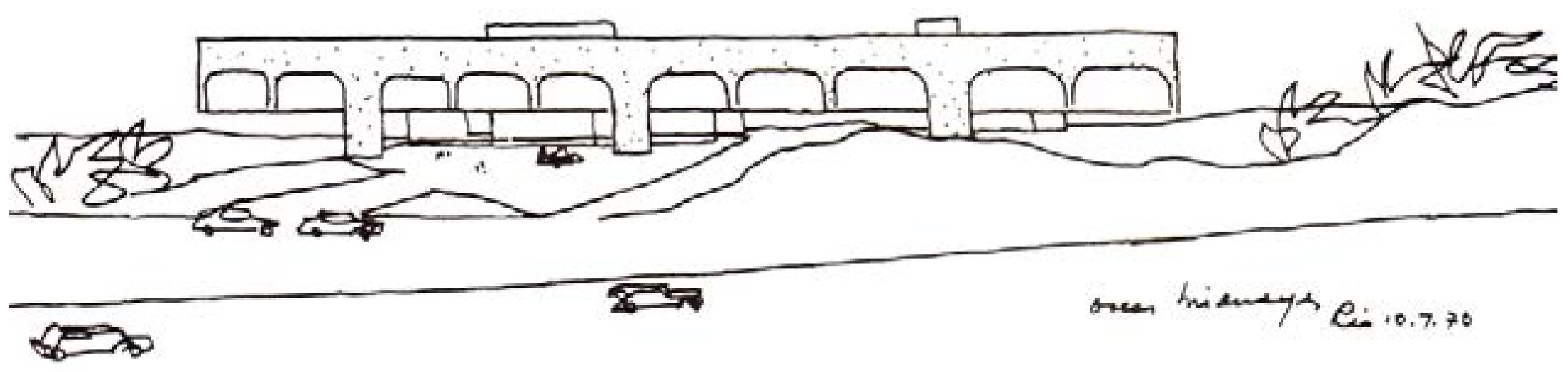

Figura 9. La sede de FATA Engineering, en las afueras de Torino.

Fuente: Archivo Fundación Niemeyer. 
El tema de la caja suspendida asume una más reciente declinación en el Edificio de oficinas del Memorial de América Latina en São Paulo, 1987. El cofre suspendido está sostenido por dos soportes exteriores en correspondencia de los lados cortos del rectángulo en planta. Las columnas no penetran el volumen: son independientes y sostienen la jácena desde la cual están colgados los forjados de los varios niveles. El edificio, de lenguaje hiperminimalista, desarrolla el tema de la dicotomía entre elementos portantes y portados gracias al auxilio de los progresos tecnológico de la ingeniería brasileña y el uso expresivo y contrastante de los colores: negro en el vidrio de las paredes de la caja, blanco en la estructura vertical de la caja, en la jácena y en las paredes laterales (Figura 10).
Esta obra ejemplifica de manera clara y didáctica la reducción del lenguaje formal del maestro, típica de varios proyectos de los años ochenta y noventa. Parece que haya la precisa voluntad de reinterpretar el lema de Mies "Menos es más", buscando lo esencial en la geometría y en la forma: de esta forma el volumen puro se podría relacionar a una escultura minimalista de Donald Judd, o de Sol Lewitt.

El uso del color tiene en este sentido una enérgica función unificadora. Los detalles arquitectónicos, así como los marcos del curtain wall y el contraste cromático entre materiales de diferentes tipos, tienden a desaparecer. Lo mismo acontece, paradójicamente, con los sistemas de quiebra soles.

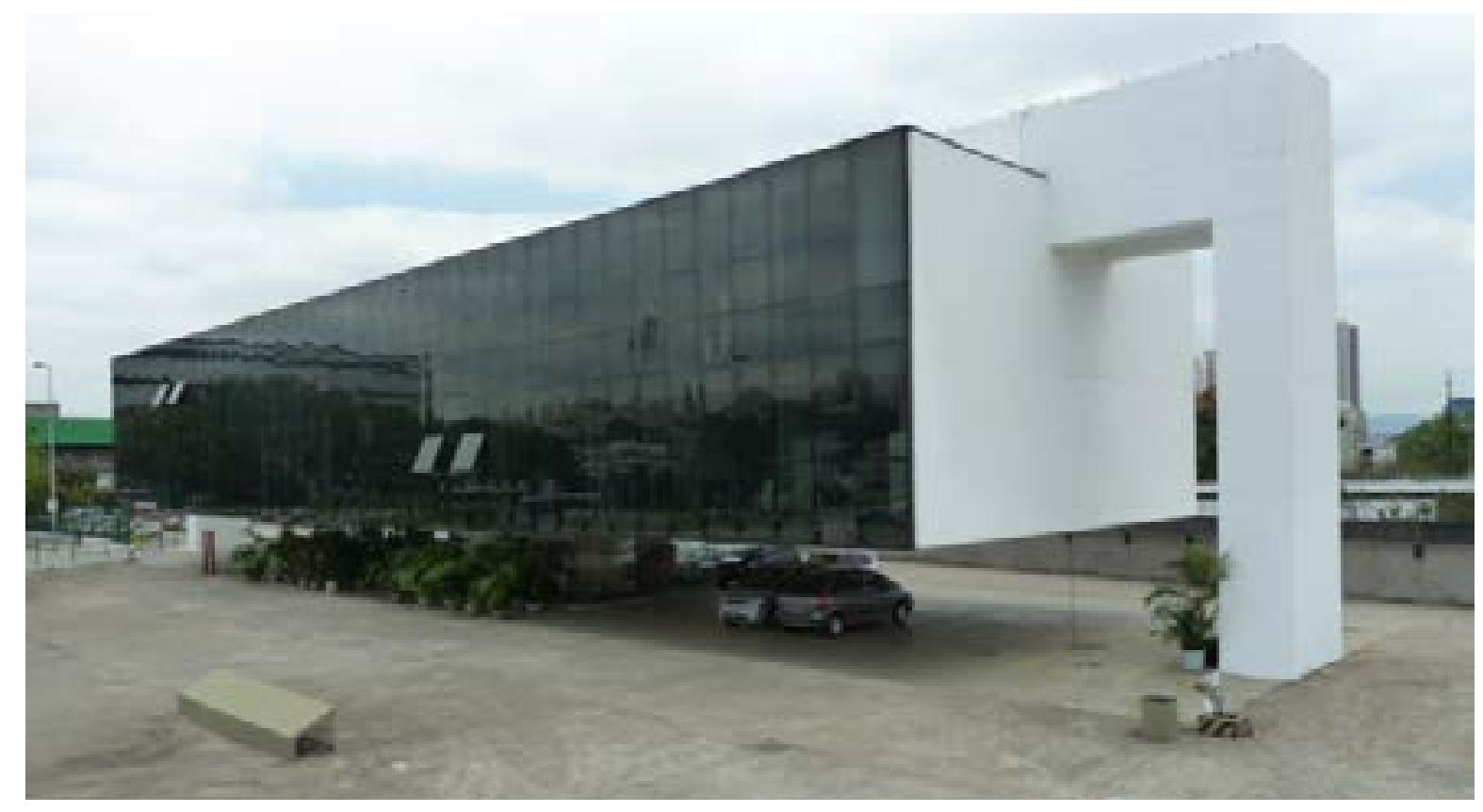

Figura 10. El edificio de oficinas en el Memorial de América Latina (São Paulo).

Fuente: Foto de Luca Bullaro. 
Después de varios decenios de búsqueda paciente y de múltiples ensayos en el uso de varios sistemas de protección solar, aparece contundente la decisión de renunciar a este sabio conjunto de pieles bioclimáticas en favor de cristales de tonalidad oscura que no permiten el paso de los rayos ultravioletas a las estancias interiores.

Explicitando la dicotomía y la tensión entre elementos soportados y de soporte, el cofre se vuelve totalmente minimalista: un volumen homogéneo y purísimo bajo la intensa luz tropical. Un homenaje al maestro Le Corbusier, declinado respecto al clima tropical de Brasil y la voluntad de una absoluta pureza.

\section{Conclusiones}

A partir del análisis de este conjunto de proyectos que obedecen a principios formales, plásticos y geométricos de matriz similar, se han individuado, en el transcurso de la investigación, algunas diferentes tipologías en el marco general de los "prismas minimalistas"; y se propuso una clasificación:

1. El prisma puro, que deriva de algunos proyectos de Le Corbusier, como por ejemplo el Pabellón de Suiza en Paris;

2. El prisma monumental, de doble fachada, que confiere un aura simbólica, y que al mismo tiempo protege del sol y de la lluvia;

3. La caja suspendida, que se basa en un sistema macro estructural de pilastras y jácenas dispuestas en el último andar, al cual se suspenden los forjados intermedios;
4. La caja suspendida mediante arcos en hormigón armado que concatenan elementos estructurales verticales y horizontales, generando una continuidad morfológica entre elementos que trabajan a compresión y otros que trabajan a tensión.

\section{La caja hiper-minimalista.}

La evolución de este tipo, el prisma purista, parece, en síntesis, dividirse en dos apéndices fundamentales: el primero persigue el tema de la monumentalidad moderna, que exteriormente se evidencia con el uso de arcos en concreto de diferentes formas y ritmos; el segundo apéndice tipológico se proyecta hacia una poética que se podría definir "hiper-minimalista".

De este segundo apéndice es ejemplo contundente el bloque suspendido de las oficinas del Memorial de Latinoamerica, en Sao Paulo -inaugurado en 1989- que expresa una reducción contundente de los elementos formales y arquitectónicos, acompañada por una fuerte alternancia cromática y una ausencia total de quiebra-soles, sustituidos por vidrios muy oscuros. En esta obra:

- No hay columnas sino dos pilotis de gran tamaño colocados externamente al perímetro de la caja.

- No hay ventanas, sino un uniforme pan de verre en el cual se dificulta la individuación de los marcos metálicos: siendo el color negro de los marcos similar a la tonalidad de los vidrios.

- No se perciben tenues variaciones cromáticas, sino contrastes entre blanco y negro. 
Este ejemplo ayuda a comprender la voluntad de Niemeyer de reducir, y de forma contundente, los elementos arquitectónicos - a menudo de difícil recognoscibilidad - con el fin de plasmar un objecto puro que posee similitudes con las esculturas del movimiento Minimalista.

Los análisis comparados entre edificios que pertenecen a una misma familia conceptual y morfológica son útiles para proponer ulteriores clasificaciones tipológicas y para analizar también las transformaciones de lenguaje que Niemeyer actúa a lo largo de su extenso recorrido profesional.

Este tipo de reflexiones analíticas puede abrir nuevas pistas para ulteriores investigaciones, $\mathrm{y}$ también para estudios comparados sobre la obra de uno de los más importantes arquitectos de América Latina que - como afirma Oriol Bohigas (2012)— "ha introducido unas modificaciones $\mathrm{y}$ unos cambios de actitud, que caracterizan unas fórmulas nuevas (...) que han marcado un paso importante en la evolución de la arquitectura contemporánea".

\section{REFERENCIAS}

Bohigas, O. (diciembre 6, 2012). Ética y política de la arquitectura. El Pais. Disponible en https://elpais.com/cultura/2012/12/06/actualidad/1354824125_469043.html

Bullaro, L. (2014). Moderno y tropical: la reinterpretación de los principios lecorbuserianos en las primeras obras de Oscar Niemeyer. DEARQ, (15), 36-51.
Costa, L. (1961). Relatório Lucio Costa. Arquitetura e engenharia. Belo Horizonte, 61(3), $17-20$.

Frampton, K. (2009). Homenagem a Niemeyer. En, R. Segre, Tributo a Niemeyer (pp. 2627). Rio de Janeiro: Viana.

Harris, E. (1987). Le Corbusier: riscos brasileiros. São Paulo: Nobel.

Niemeyer, O. (2012). Il mondo è ingiusto. Milano: Mondadori.

Niemeyer, O. (2000). Minha arquitectura. Rio de Janeiro: Revan.

Niemeyer, O. (1978). La forma nell'architettura. Milano: Mondadori.

Niemeyer, O. (1960). Minha experiência em Brasília. Módulo, 3(18), 11-16.

Niemeyer, O. (1958). Depoimento. Módulo, (9), 3-6.

Papadaki, S. (1954). Oscar Niemeyer. [Work in progress]. New York: Reinhold.

Segre, R. (2012). Oscar Niemeyer. Tipologias e liberdade plástica. Arquitextos, 13(151). Disponible en https://www.vitruvius.com.br/ revistas/read/arquitextos/13.151/4604

Serapiao, F. (2005). Oscar Niemeyer: Auditório Ibirapuera. SP. Projeto, (309). Disponible en: https://www.arcoweb.com.br/projetodesign/arquitetura/oscar-niemeyer-auditoriosao-14-12-2005

Verde, R. (2012). Oscar Niemeyer. Da critica alheia à teoria própria. Arquitextos, 13(151.04). Disponible en https://www.vitruvius.com.br/ revistas/read/arquitextos/13.151/4608 
Wainwright, O. (2012). Oscar Niemeyer: architects and critics pay tribute. The Guardian. Disponible en https://www.theguardian.com/ artanddesign/architecture-design-blog/2012/ dec/07/oscar-niemeyer-tributes-architectscritics

Zevi, B. (1982). Storia dell'architettura moderna. Bologna: Zanichelli.

Zevi, B. (1971). Brasilia. [Cronache di architettura I]. Roma: Laterza.
Luca Bullaro: Doctor en Proyectos Arquitectónicos de la Univesitát degli Studi di Palermo (España). Magister de la Universitat Politècnica de Catalunya (España). Arquitecto de la Univesitát degli Studi di Palermo (España). Profesor asociado a la facultad de Arquitectura de la Universidad Nacional (Colombia). 